\title{
Growth of Large Size Lithium Niobate Single Crystals of High Quality by Tilting-mirror-type Floating Zone Method
}

\author{
Abdur Razzaque Sarkera, * * \\ ${ }^{a}$ Center for Crystal Science and Technology, University of Yamanashi, 7-32 Miyamae, \\ Kofu, Yamanashi 400-8511, Japan. \\ ${ }^{b}$ Department of Physics, University of Rajshahi, Rajshahi 6205, Bangladesh.
}

Received: July 22, 2015; Revised: January 26, 2016; Accepted: February 9, 2016

\begin{abstract}
Large size high quality $\mathrm{LiNbO}_{3}$ single crystals were grown successfully by tilting-mirror-type floating zone (TMFZ) technique. The grown crystals were characterized by X-ray diffraction, etch pits density measurement, Impedance analysis, Vibrating sample magnetometry (VSM) and UV-Visible spectrometry. The effect of mirror tilting during growth on the structural, electrical, optical properties and defect density of the $\mathrm{LiNbO}_{3}$ crystals were investigated. It was found that the defect density in the crystals reduced for tilting the mirror in the TMFZ method. The chemical analysis revealed that the grown crystals were of high quality with uniform composition. The single crystals grown by TMFZ method contains no low-angle grain boundaries, indicating that they can be used for high efficiency optoelectronic devices.
\end{abstract}

Keywords: Lithium niobate, Laue technique, Floating zone technique, Defects, Optoelectronic materials

\section{Introduction}

Lithium niobate, $\mathrm{LiNbO}_{3}(\mathrm{LN})$ is an important ferroelectric material that has found applications in a wide range of optoelectronic devices, laser physics, holography, and acoustics ${ }^{1,2}$. LN single crystals are important materials for optical waveguides, mobile phones, piezoelectric sensors, optical modulators, photorefractive devices, optical frequency multipliers, surface acoustic wave devices and various linear and non linear optical applications ${ }^{3-7}$. It has negative uniaxial birefringence which depends slightly on the stoichiometry and strongly on the crystal structure and temperature ${ }^{8}$. $\mathrm{ALiNbO}_{3}$ crystal is one of the most promising materials in optoelectronics owing to its excellent optical properties ${ }^{9,10}$. However, the optical properties of a $\mathrm{LiNbO}_{3}$ crystals have not been widely utilized, because it was not easy to overcome some disadvantages in the crystal growth as i) low thermal shock resistance ii) numerous light scattering centers and iii) local fluctuation of the refractive indices in the grown crystals ${ }^{11}$

In general, $\mathrm{LiNbO}_{3}$ crystal is grown by the Czochralski (CZ) method in which compositional variation along the growth axis occurs in the crystals that affects several physical properties ${ }^{2} . \mathrm{LiNbO}_{3}$ single crystals of $20 \mathrm{~mm}$ in diameter and $75 \mathrm{~mm}$ in length were grown by Bermudez et al., using $\mathrm{CZ}$ technique from a congruent melting composition, $\mathrm{Li} / \mathrm{Nb}=48.6: 51.4^{12}$. In the $\mathrm{LiNbO}_{3}$ single crystals grown by $\mathrm{CZ}$ method, some defects like cation vacancies are always present. ${ }^{13}$. Moreover, $\mathrm{CZ}$ grown crystals may contain some dislocations and subgrain boundaries. These defects seriously affect many physical properties and limit practical applications ${ }^{3}$. All applications of $\mathrm{LiNbO}_{3}$ crystals require

*e-mail : razzaque_ru2000@yahoo.com high optical quality and control of composition. Therefore, both large size and defect free $\mathrm{LiNbO}_{3}$ single crystals are needed for design of various optical devices. Iyi et al., used a conventional Infrared heating floating zone (IR-FZ) method to grow $\mathrm{LiNbO}_{3}$ single crystals with improved quality and homogeneous composition but it was difficult to obtain crystals of large diameter ${ }^{14}$.

Floating zone (FZ) method is a powerful technique for growth of single crystals without contaminations because it is a crucible free zone melting method. In the conventional Infrared heating (IR-FZ) method, crystal diameter is limited by the shape of the molten zone. The shapes for both melt-feed and melt-crystal interfaces are convex towards melt in FZ method. A more convex crystal-melt interface might cause an easier drop of the melt, especially during the growth of the crystals with a larger diameter because the unexpected contact of the feed rod and the grown crystal in the molten zone during growth is a serious problem in growing a large-diameter crystal ${ }^{15}$. Therefore, the control of the convexity is one of the important issues to overcome the limitations. A slightly convex interface towards melt is better for improving the quality of crystals ${ }^{7}$. We reported that the interface shape can be controlled by tilting the mirror in the tilting-mirror-type floating zone (TMFZ) method as shown in fig. $1^{16}$. It was revealed that the quality of the TMFZ-grown crystals increases with increase of tilting angle ${ }^{17}$ and growth diameter ${ }^{18}$.

In this study, we try to grow high quality $\mathrm{LiNbO}_{3}$ single crystal by TMFZ method for optical device applications. The TMFZ method is an outstanding way of growing high quality single crystals especially for oxides. The $\mathrm{LiNbO}_{3}$ 
single crystals were grown at $\theta=20$ degree by this method because it was reported to be suitable for growth of high quality single crystals ${ }^{17}$. This report deals with the merit of TMFZ method for growing large size $\mathrm{LiNbO}_{3}$ crystals with reduced dislocations and subgrain boundaries. Subgrain boundaries are one of the main causes of the local fluctuations of refractive indices ${ }^{11}$. The main aim of this work is the growth of optically homogeneous single domain $\mathrm{LiNbO}_{3}$ crystals for high performance device applications.

\section{Experiment}

Anew tilting-mirror-type image furnace (Crystal Systems Corporation; model TLFZ-4000-H -VPO ) was developed for our growth experiment. The tilting angle $(\theta)$, as shown in fig. 1 , can be changed to up to $30^{\circ}$ by a motor drive control. High-purity ( $>99.99 \%$ ) powders of $\mathrm{Li}_{2} \mathrm{CO}_{3}$ and $\mathrm{Nb}_{2} \mathrm{O}_{5}$ were used as the starting material for feed preparation. $100 \mathrm{~g}$ powder (48.6 mol\% $\left.\mathrm{Li}_{2} \mathrm{CO}_{3}+51.4 \mathrm{~mol} \% \mathrm{Nb}_{2} \mathrm{O}_{5}\right)$ was ball milled and mixed powder was calcined at $900{ }^{\circ} \mathrm{C}$ for $12 \mathrm{~h}$ in air. The powder was grinded and calcined again under the same conditions. Then the powder was grinded again and filled into a rubber tube using a long glass bar, and shaped into a rod. After sealing, the rod was pressed up to $3 \times 10^{8} \mathrm{~Pa}$ using a cold isostatic pressing machine (Nikkiso Co., Ltd.: model CL3-22-60). The pressed rods were typically $18-20 \mathrm{~mm}$ in diameter and $\sim 150 \mathrm{~mm}$ in length. A hole on the rod was drilled and the rod was tied with a Pt-Rh wire for hanging and then sintered at $1100{ }^{\circ} \mathrm{C}$ for $12 \mathrm{~h}$ in air. After sintering, the feed rod became $15-17 \mathrm{~mm}$ in diameter and $\sim 130 \mathrm{~mm}$ in length.

The applied conditions for growth experiment were a growth rate of $3 \mathrm{~mm} / \mathrm{h}$, upper shaft rotation of $10 \mathrm{rpm}$, lower shaft rotation of $30 \mathrm{rpm}$, a growth direction of $<100>$ using a seed crystal. The crystals were grown within oxygen pressures of $3 \mathrm{~atm}$. To enlarge the crystal diameter, the feeding speed was gradually increased 3 to $9 \mathrm{~mm} / \mathrm{h}$. In case of TMFZ furnace, the tilting angle, $\theta$ was $20^{\circ}$. It has been reported that this tilting condition is the best for growth of good quality large diameter single crystals with most stabilized molten zone ${ }^{1,8}$.

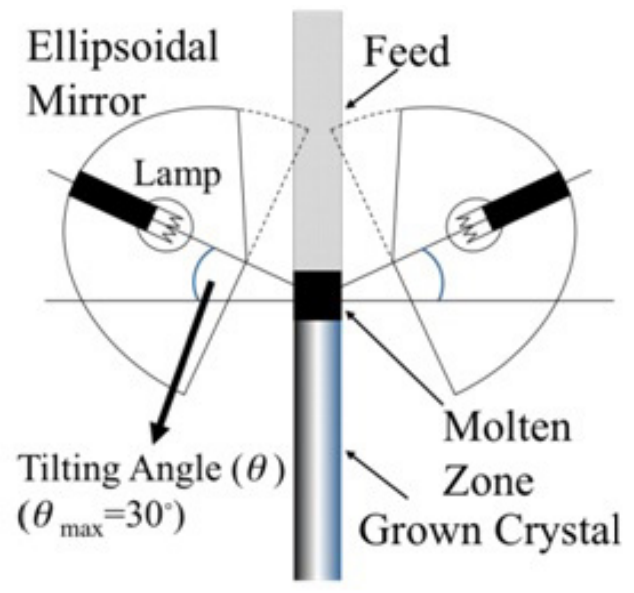

Fig. 1. Schematic illustration of the tilting mirror type image furnace. The definition of tilting angle $(\theta)$ is given.
The grown crystal was checked as single crystal by Laue technique using Rigaku X-ray diffractmeter. The crystals were cut perpendicular to the growth direction and parallel to the growth direction. Then the surface was polished like mirror. The polished surfaces were observed by optical microscope (Olympus U-MSSPG Japan) to investigate the phase purity and optical quality. The polished samples were soaked in a mixture $\mathrm{HF}$ and $\mathrm{HNO}_{3}$ solutions (1:2 in volume) for $1 \mathrm{~h}$ at $100{ }^{\circ} \mathrm{C}$ to etch their surfaces for the purpose of observation of domain structure. The chemical composition was checked by Electron probe micro analyzer (EPMA) (JEOL: model JXA-8200). For quantitative analysis, a $\mathrm{LiNbO}_{3}$ single crystal was used as standard to characterize the quality and composition of the grown crystals. The Agilent Precision Impedance Analyzer (Agilent technologies, Model: 4294A Japan) was used for measurements of frequency dependences ac conductance, impedance, dielectric constant and capacitance. The temperature dependence magnetic susceptibility was measured by Quantum Design Dynacool physical properties measurement system (PPMS).

\section{Results and discussions}

\subsection{Structural properties and compositions}

Lithium niobate has a trigonal crystal structure and is characterized by large pyroelectric, piezoelectric, electro-optic and photoelastic coefficients ${ }^{19}$. Although $\mathrm{LiNbO}_{3}$ single crystal is important in broad areas of technological applications, the details of the physical properties and crystal structure are not readily available in literature. A bulk single crystal of $\mathrm{LiNbO}_{3}$ is shown in fig. 2(a) was grown using conventional IR-FZ method along the Z-axis in the oxygen pressure of $0.2 \mathrm{MPa}$. The $\mathrm{LiNbO}_{3}$ single crystal shown in fig. 2(b) was grown in the same conditions using TMFZ method to compare the crystal quality. The grown crystals were inclusion free. The grown crystals were cut perpendicular to the c-axis and Laue X-ray diffraction was taken to check the crystalinity and crystal structure. Fig. 3 shows the Laue $\mathrm{x}$-ray diffraction pattern of TMFZ grown $\mathrm{LiNbO}_{3}$ single crystal. The Laue analysis of X-ray diffraction pattern of the crystals reveals that they were single crystals and their growth directions were the [001] direction parallel to the $c$-axis. This analysis also showed that the crystal structure was trigonal with hexagonal symmetry.

The powder samples of $\mathrm{LiNbO}_{3}$ after sintering and crystal growth were characterized at the room temperature by a Rigaku Mutiflex diffractometer range from $2 \theta=5^{\circ}$ to $85^{\circ}$ with $\mathrm{CuK}_{\alpha}$ radiation $(\lambda=1.5418 \AA)$, at $40 \mathrm{KV}$ and $30 \mathrm{~mA}$. Fig. 4(a) shows the $X$-ray diffraction pattern of the precursor

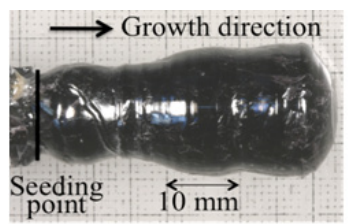

(a)

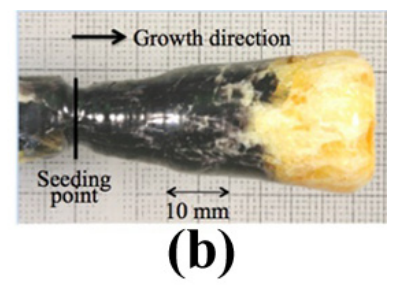

Fig. 2. Optical photographs of $\mathrm{LiNbO}_{3}$ single crystals grown by (a) Conventional FZ and (b) TMFZ method. 


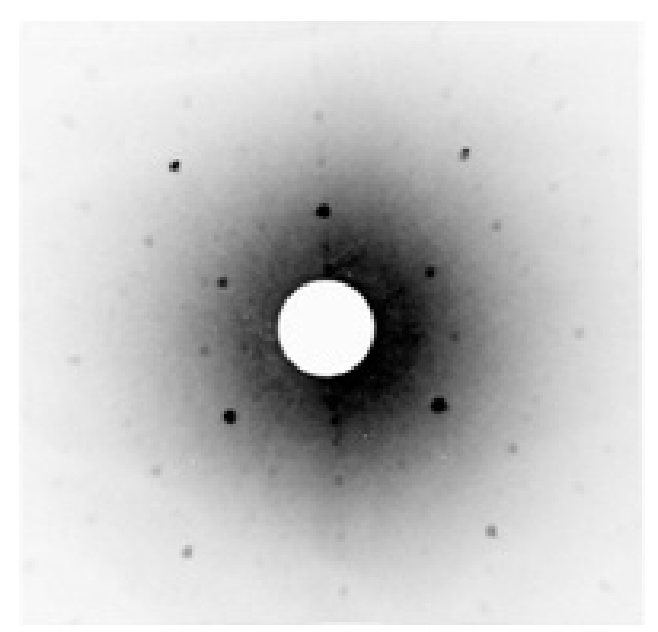

Fig. 3. X-ray diffraction Laue photographs of $\mathrm{LiNbO}_{3}$ single crystal grown by TMFZ method.

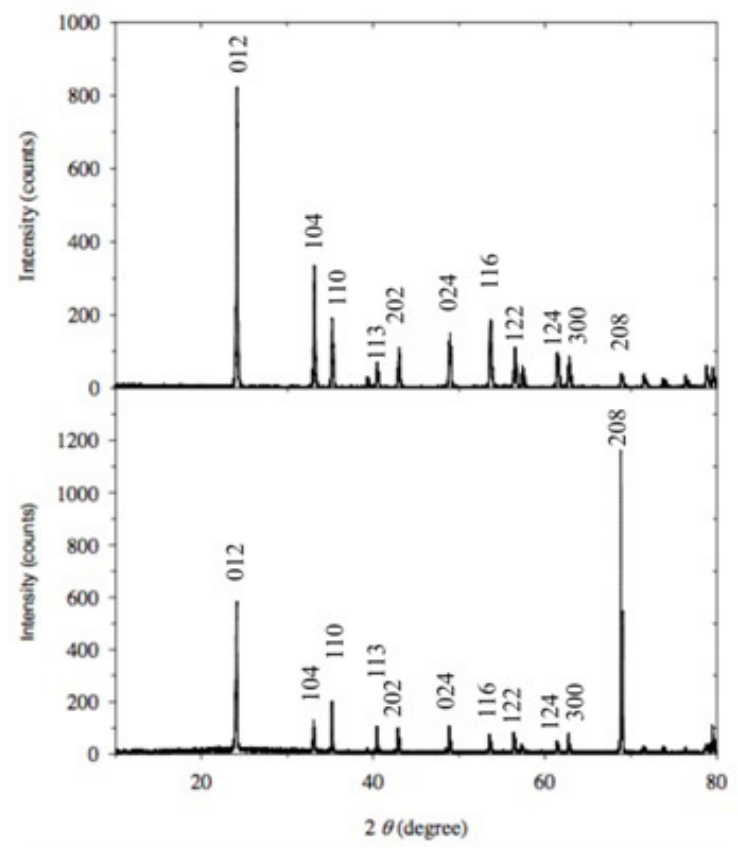

Fig. 4. X-ray diffraction pattern of (a) $\mathrm{LiNbO}_{3}$ crystalline powder after sintering (b) $\mathrm{LiNbO}_{3}$ single crystal grown by TMFZ method. after calcinated at $900{ }^{\circ} \mathrm{C}$ and fig. 4(b) shows that of $\mathrm{LiNbO}_{3}$ single crystals grown by TMFZ method. It is clearly observed that single crystal of lithium niobate was obtained from the polycrystalline precursor without any intermediate phase. All diffraction peaks in the curve were assigned as single phase $\mathrm{LiNbO}_{3}$. The unit cell refinement was accomplished using XRD data and CellCall software. Typical XRD patterns of calcinated powder and single crystal of $\mathrm{LiNbO}_{3}$ reveal that all these materials have trigonal crystal structure with space group R3c. The $\mathrm{LiNbO}_{3}$ bulk single crystals were also grown by Chiang and Chen using Czochralski (CZ) method for characterizations ${ }^{20}$. The Table 1 shows the comparison of lattice parameters of FZ and $\mathrm{CZ}$ grown bulk single crystals and sintered ceramic powders of $\mathrm{LiNbO}_{3}$. The values in the bracket indicate the error in the calculated values.

The prepared samples were identified as highly crystalline and homogeneous by indexing these XRD patterns using JCPDS data No. 44-0145. Here the sharp peaks indicate the good crystallinity. In the XRD pattern the impurity phase peaks of $\mathrm{Nb}_{2} \mathrm{O}_{5}$ and $\mathrm{Li}_{2} \mathrm{CoO}_{3}$ are not present, so pure phase solid is obtained. The good crystallinity of $\mathrm{LiCoO}_{2}$ crystals would improve the efficiency of the optical devices.

In order to get physical insight of the chemical bonding and phase formation, we have used FTIR spectroscopy. FTIR spectroscopic technique is powerful for investigating the local structure and cation environment in oxides ${ }^{22,23}$. Fig. 5 shows the Fourier transform infrared (FTIR) spectrum of $\mathrm{LiNbO}_{3}$ single crystals. The main spectrum of $\mathrm{LiNbO}_{3}$ contains the following main absorption bands: 228, 280, 1078 and $2799 \mathrm{~cm}^{-1}$. These results confirm the XRD observations showing that the vibration bands for precursors vanished and the vibration bands for the oxide network developed. In the far-infrared region, we observed the single well-resolved band at $228 \mathrm{~cm}^{-1}$. This sharp band in $\mathrm{LiNbO}_{3}$ is assigned due to an asymmetric stretching motion of the $\mathrm{LiO}_{6}$ octahedra ${ }^{24}$. It is the unique finger print of the Li site occupancy in the trigonal structure. There is a sharp medium absorption peaks around $1078 \mathrm{~cm}^{-1}$, which is due to the vibrations of the lithium niobate crystal lattice. The results of FTIR analysis were good agreement with XRD results. Therefore, we conclude that the single phase $\mathrm{LiNbO}_{3}$ single crystals of $25 \mathrm{~mm} \phi$ are obtained at optimum conditions.

\subsection{Phase purity and defect analysis}

Fig. 6 shows the compositional profile of TMFZ grown $\mathrm{LiNbO}_{3}$ crystals along growth direction and diameter. Fig. 6(a) shows that the compositions of lithium, niobium

Table 1. The comparison of lattice parameters of bulk single crystals and ceramics

\begin{tabular}{|c|c|c|}
\hline Sample & Lattice parameters & Reference \\
\hline \multirow[b]{2}{*}{$\mathrm{CZ}$ grown $\mathrm{LiNbO}_{3}$ bulk single crystals } & $\begin{array}{l}a=b=5.149 \AA c=13.894 \AA \\
V=318.450 \AA^{3}\end{array}$ & 20 \\
\hline & $\begin{array}{l}a=b=5.150 \AA c=13.864 \AA \\
V=318.449 \AA^{3}\end{array}$ & 21 \\
\hline FZ grown $\mathrm{LiNbO}_{3}$ bulk single crystals & $\begin{array}{l}a=b=5.101 \AA(\sim 0.0092) c=13.831 \AA(\sim 0.026) \\
V=311.686 \AA^{3}(\sim 1.28)\end{array}$ & This work \\
\hline $\mathrm{LiNbO}_{3}$ ceramic powder & $\begin{array}{l}a=b=5.082 \AA(\sim 0.0118) c=13.830 \AA(\sim 0.034) \\
V=309.362 \AA^{3}(\sim 1.63)\end{array}$ & This work \\
\hline
\end{tabular}




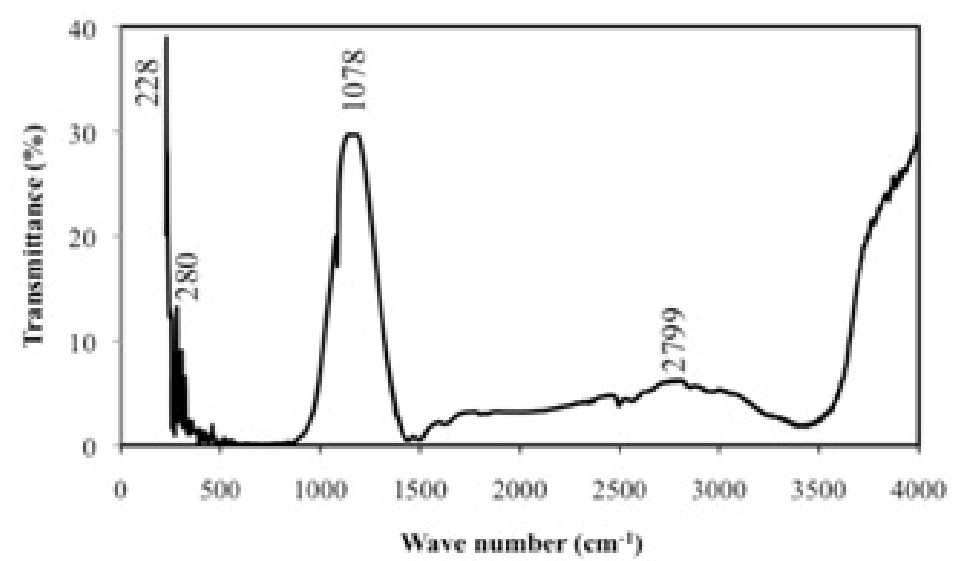

Fig. 5. FT-IR spectrum of $\mathrm{LiNbO}_{3}$ single crystal grown by TMFZ method.

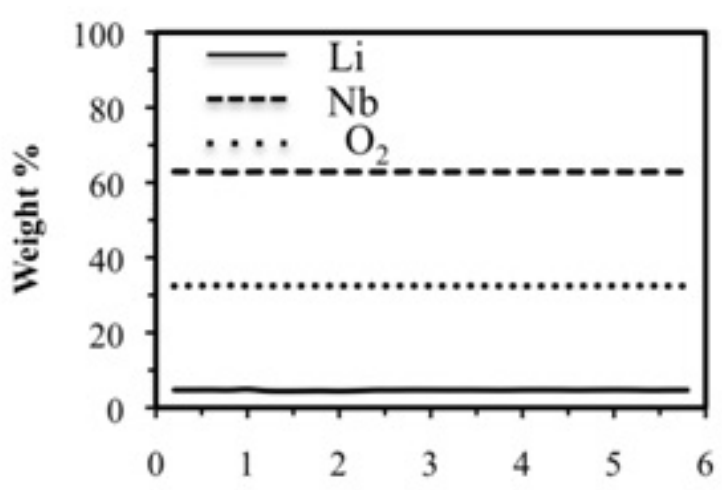

Growth length $(\mathrm{mm})$

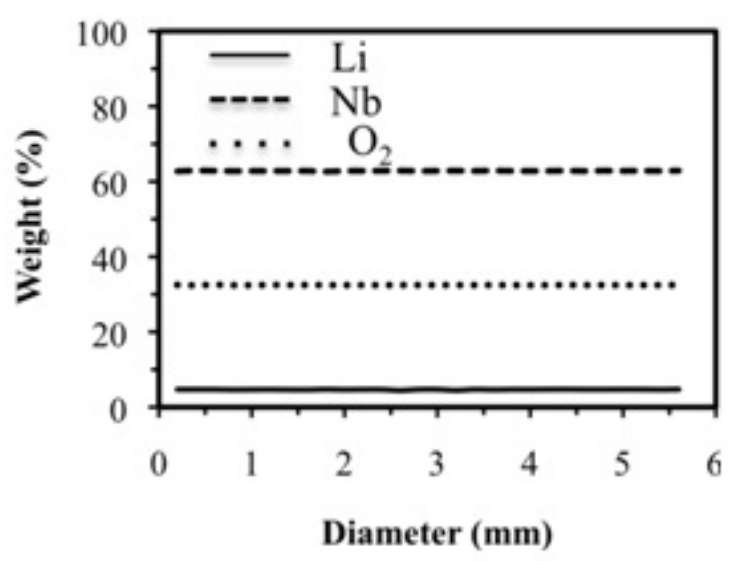

Fig. 6. Weight percentage of lithium, niobium and oxygen in $\mathrm{LiNbO}_{3}$ single crystals along (a) growth direction and (b) radial direction (diameter).

and oxygen are uniform along growth direction. There are no significant variations of weight percent of constituent elements through growth length. Fig. 6(b) shows that the compositions of lithium, niobium and oxygen are uniform along cross-section. There are no significant variations of weight percent of constituent elements along growth diameter. These results showed that $\mathrm{LiNbO}_{3}$ grown crystal were homogeneous in composition. The chemical analysis by EPMA revealed that there were no secondary phases produced in the crystal during growth. Hence single phase $\mathrm{LiNbO}_{3}$ crystals was obtained by TMFZ method.

The crystals grown by conventional IR-FZ and TMFZ were characterized by determining etch pit density (EPD) because Hirthe et al. showed that etch pits are known to be a good measure of the dislocation density ${ }^{25}$. Kinoshita et al., showed that etch pits arise in the presence of low-angle grain boundaries ${ }^{26}$. As the presence of defects like dislocations, grain boundaries etc. degrades the quality of the grown crystals, we measure the etch pits to clarify effect of mirror tilting on crystal quality. Fig. 7 shows optical microphotographs of the samples on the (100) surface after chemical etching. The observed etch pits are clearly few in the crystal grown by conventional floating zone method. On the other hand
TMFZ grown crystal was almost free of etch pits. These results reveal that $\mathrm{LiNbO}_{3}$ grown by TMFZ method was defect free high quality single crystal. In the previous study, we showed that the defects in the rutile single crystals were reduced by tilting the mirrors in the TMFZ method ${ }^{17}$. This study also established the importance of TMFZ method to grow high quality single crystals.

\subsection{Electronic and magnetic properties}

Electrical conductance of the samples were measured by impedance analyzer, where the signal frequency was varied from $100 \mathrm{~Hz}$ to $1 \mathrm{M} \mathrm{Hz}$ with applied oscillating voltage of $300 \mathrm{mV}$. All measurement has been carried out at room temperature. The frequency dependence electrical conductance $\mathrm{LiNbO}_{3}$ single crystal grown by TMFZ method is shown in the fig 8(a). The electrical conductance increases rapidly with frequency at the lower frequency range. The conduction in the material requires the hopping of charges between the allowed sites. Fig. 8(a) shows that low frequency region (up to $200 \mathrm{kH}$ ) is almost frequency independent and that of high frequency is dispersive region. Low frequency response indicate the long range transport of activated charges (dc conductivity) with regard to the applied electric field. 
The dispersive region at high frequencies can be explained with respect to the diffusion controlled relaxation model.

Impedance spectroscopy is a relatively new and powerful method of characterizing many of the electrical properties of electrolyte materials and their interfaces with electronically conducting electrodes. Fig. 8(b) shows the frequency dependence impedance of the $\mathrm{LiNbO}_{3}$ single

(a)

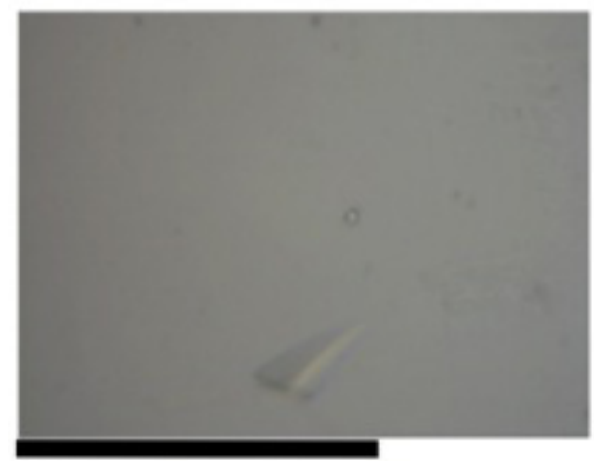

$100 \mu \mathrm{m}$ crystals grown by TMFZ method. Impedance has been measured by impedance analyzer, where frequency was within $100 \mathrm{kHz}-1 \mathrm{MHz}$ with applying oscillating voltage of $300 \mathrm{mV}$. The measurement has been carried out at room temperature. In the impedance measurements a sinusoidal potential is applied and the impedance and phase shift of the current are measured. At higher frequencies (above $600 \mathrm{KHz}$ ) $\mathrm{Z}$ is (b)

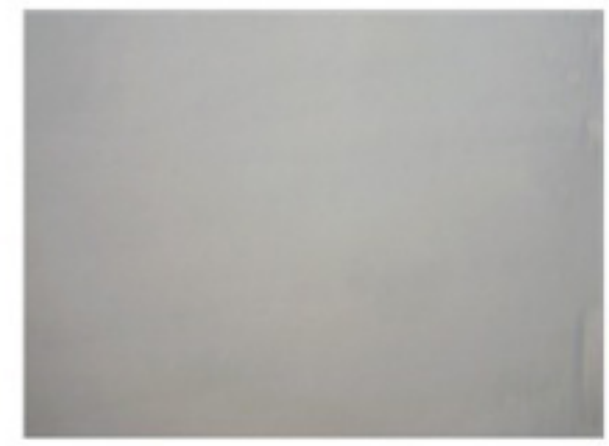

Fig. 7. Optical microphotographs of etch pits on (100) of $\mathrm{LiNbO}_{3}$ single crystals grown by (a) conventional FZ (b) TMFZ method.
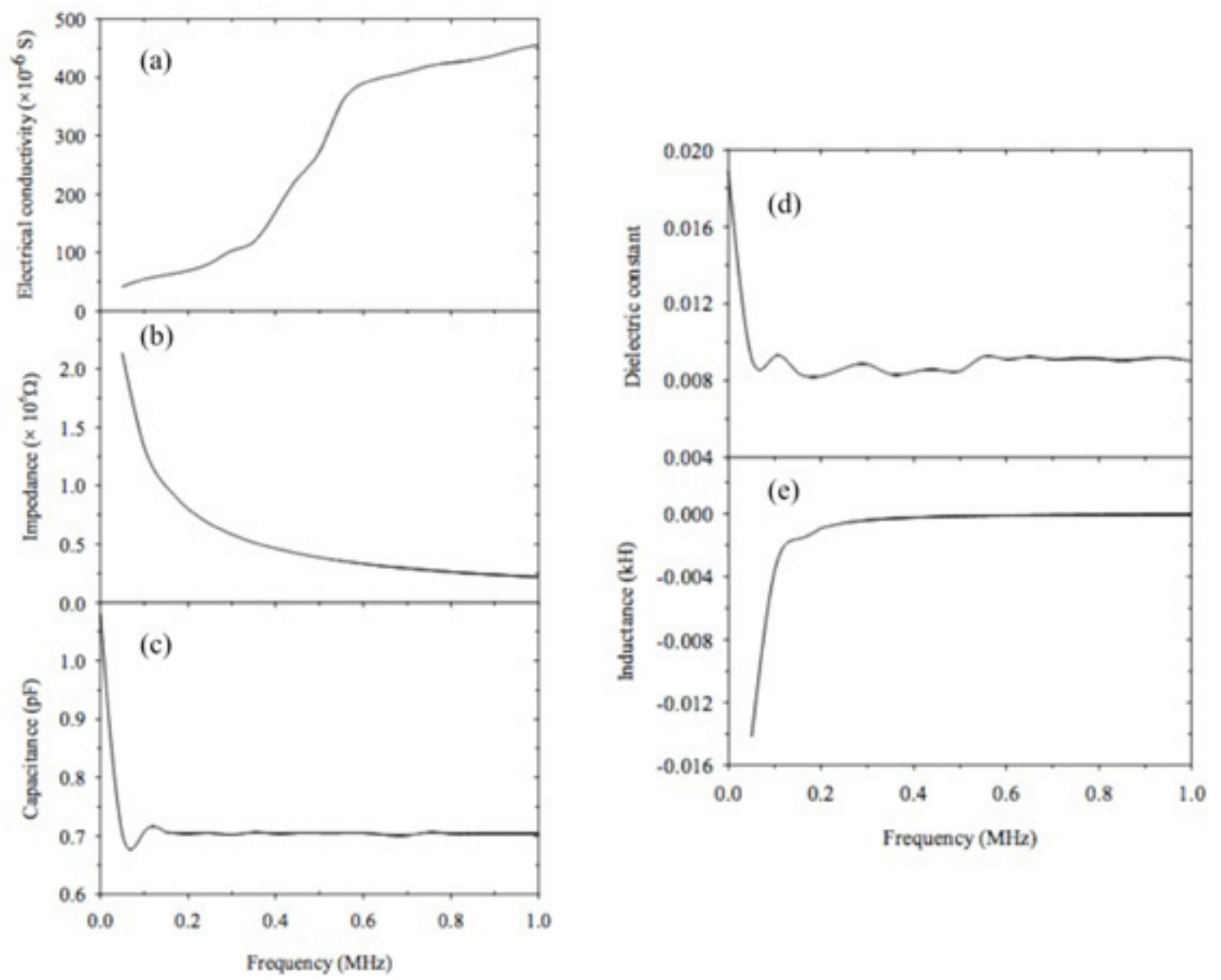

Fig. 8. Frequency dependence (a) Electrical conductivity (b) Impedance (c) Capacitance (d) Dielectric constant and (e) Inductance of $\mathrm{LiNbO}_{3}$ single crystals grown by TMFZ method. 
almost independent of frequency, which is attributed to the resistance effect. In the low frequency ranges, $\mathrm{Z}$ considerably decreases as the frequency increases.

The frequency dependent capacitances of samples were measured by impedance analyzer within the frequency range $100 \mathrm{~Hz}$ to $1 \mathrm{MHz}$ at room temperature and oscillating voltage $300 \mathrm{mV}$ was applied. Fig. 8(c) shows the frequency dependence capacitance of $\mathrm{LiNbO}_{3}$ single crystals grown by TMFZ method. At the low frequency region capacitance decreases sharply and then increases slightly with frequency. The capacitance of $\mathrm{LiNbO}_{3}$ single crystal is high at low frequency region due to contribution of all kinds of polarization at low frequency. Then the capacitance decreases with increase of frequency and finally approaches to an almost constant value above $150 \mathrm{kHz}$. This is due to the change of space charge, ionic and orientation polarization at higher frequencies ${ }^{27}$.

The dielectric property of a material is another important factor in electronic device applications. Dielectric properties of the $\mathrm{LiNbO}_{3}$ single crystal grown by TMFZ method was measured by the precision impedance analyzer. Silver paste was coated on both surface of each sample before measurement. Dielectric constant is a measure of materials ability to store electric charge. Dielectric constant also called the real part of dielectric permittivity $\left(\varepsilon^{\prime}\right)$. The frequency dependent dielectric constants of samples were measured within the range $100 \mathrm{kHz}-1 \mathrm{MHz}$ at room temperature with the oscillating voltage $300 \mathrm{mV}$. Fig. 8(d) shows the plot of dielectric constant with frequency of $\mathrm{LiNbO}_{3}$ single crystal. It is observed that the dielectric constant is increased at lower frequencies. The high dielectric constants of $\mathrm{LiNbO}_{3}$ single crystal at low-frequency range indicate the increased migration and polarization of $\mathrm{Li}^{+}$ions at electrode-electrolyte interface. The bulk polarization of the sample results from the presence of electrodes of $\mathrm{Li}^{+}$ions at electrode-electrolyte interface. The behavior of the dielectric permittivity with frequency is related to the applied field, which assists electron hopping between two different sites of the sample. At higher frequency region, the charge carriers will no longer be able to rotate sufficiently rapidly, so their oscillation will begin to lay behind this field resulting in a decrease. Generally, the relaxation phenomena in dielectric materials are associated with frequency dependent orientation polarization. At low frequency region, the permanent dipoles align themselves along the field and contribute fully to the total polarization of the dielectric. At higher frequency region, the variation in the field is very rapid for the dipoles to align themselves, so their contribution to the polarization and hence, to dielectric permittivity can become negligible.

The decrease of the dielectric constant $\varepsilon^{\prime}$ can also be explained considering the interfacial polarization. The interfacial polarization arises as a result of difference in conducting phase, but is interrupted at grain boundary due to lower conductivity. Generally in polycrystalline materials, the grain exhibits semiconducting behavior while the grain boundaries are insulators. Fig. 8(e) shows the frequency dependence self-inductance of the $\mathrm{LiNbO}_{3}$ single crystal. The sample was found to be diamagnetic at low frequency region. The inductance decreases with frequency up to $200 \mathrm{kHz}$ then became stable. The inductance is negative in the low frequency region and become zero above $500 \mathrm{kHz}$. This results shows that the $\mathrm{LiNbO}_{3}$ crystal is not magnetic in nature.
Bulk magnetic susceptibility of the sample was measured using a Quantum Design PPMS Magnetometer in a 1000 Oe applied field at temperature range $2-310 \mathrm{~K}$. The temperature dependent magnetic susceptibility in the field cooling (FC) condition of $\mathrm{LiNbO}_{3}$ single crystal grown by TMFZ method is shown in fig. 9. No hysteresis was found, to be caused by ferromagnetism, in the field dependent magnetization.

There was no impurity peaks in the plot what reveals that the sample was free from magnetic defects. The FC data exhibit a decrease in the magnetization with the temperature due to magnetic anisotropy-induced loss of long-range magnetic ordering of the material. At high temperatures (above $250 \mathrm{~K}$ ) the magnetic behavior of the sample is linear with temperature, which is typical of paramagnetic materials. At low temperatures (below 250) a departure from the linearity is observed, which indicates the presence of short-range magnetic interactions. The magnetic behavior in the single crystals of $\mathrm{LiNbO}_{3}$ under applied magnetic field is rather complex, similar to that of $\mathrm{LiCoO}_{2}$ compounds ${ }^{28}$.

\subsection{Optical properties}

Fig. 10 shows the variation of absorption with wavelength from $200 \mathrm{~nm}$ to $800 \mathrm{~nm}$. The absorption increases with increase of wavelength in the visible. There are two broad and a sharp absorption peaks in the ultraviolet region. The sharp absorption peak at $202 \mathrm{~nm}$ is very strong one. The absorption peak at $220 \mathrm{~nm}$ is very weak and that at $287 \mathrm{~nm}$ is broad

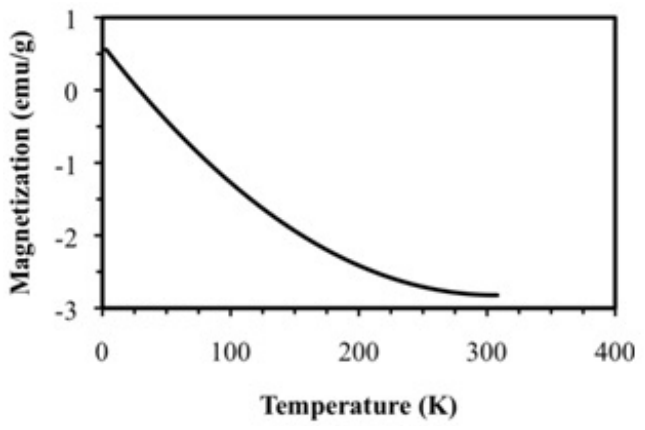

Fig. 9. Low temperature magnetization of $\mathrm{LiNbO}_{3}$ single crystals grown by TMFZ method.

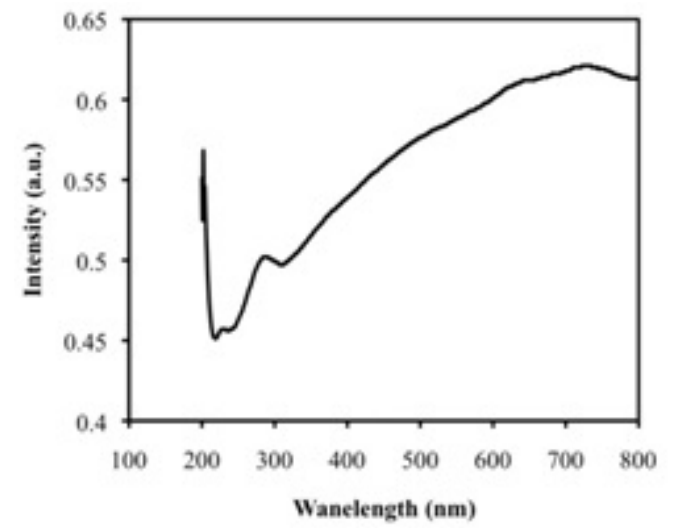

Fig. 10. The UV- Visible absorption spectrum of $\mathrm{LiNbO}_{3}$ single crystal grown by TMFZ method. 
and medium. These peaks reveal the absorption criteria of lithium niobate in the ultraviolet region. No peak was found in the visible region. This result shows that $\mathrm{LiNbO}_{3}$ is not a strong absorber of visible light. The absorptive power decreases at the end of visible region.

\section{Conclutions}

$\mathrm{LiNbO}_{3}$ is one of the most widely studied functional materials because of its diversified application. Defect free large size $\mathrm{LiNbO}_{3}$ single crystals were grown successfully by TMFZ method. Power XRD and Laue results show that the grown crystals were single phase with trigonal crystal symmetry. Comparative studies carried out on conventional floating zone and tilting mirror type floating zone grown $\mathrm{LiNbO}_{3}$ single crystals revealed that $\mathrm{FZ}$ grown single crystals are almost defect free whereas modified TMFZ grown single crystals are completely defect free. The characterization of

\section{References}

1. Polger K, Peter A, Foldvari I, Szaller ZS. Structural defects in flux-grown stoichiometric $\mathrm{LiNbO}_{3}$ single crystals. Journal of Crystal Growth. 2000;218(2-4):327-333.

2. Polger K, Peter A, Kovacs L, Corradi G, Szaller ZS. X-ray photoelectron spectroscopy investigation of substrate surface pretreatments for diamond nucleation by microwave plasma chemical vapor deposition. Journal of Crystal Growth. 1997;177(1):211-215.

3. Sun DL, Hang Y, Zhang LH, Luo GZ, Zhu SN, Lim PK, et al. Growth of near-stoichiometric $\mathrm{LiNbO}_{3}$ crystals and $\mathrm{Li}_{2} \mathrm{O}$ contents determination. Crystal Research \& Technology. 2004;39(6):511515. http://dx.doi.org/10.1002/crat.200310219

4. Tsukuda T, Kakinoki K, Hosawa M, Imaishi N, Shimamura K, Fukuda T. Numerical and experimental studies on crack formation in $\mathrm{LiNbO}_{3}$ single crystal. Journal of Crystal Growth. 1997;180(3-4):543-550.

5. Evlanova NF, Naumova II, Chaplina TO, Blokhin SA, Lavrishchev SV. Periodically poled $\mathrm{Y}: \mathrm{LiNbO}_{3}$ single crystal: impurity distribution and domain wall location. Journal of Crystal Growth. 2001;223(1-2):156-160. doi:10.1016/S0022-0248(00)01001-0

6. Naumova II, Evlanova NF, Gilko OA, Lavrishchev SV. Study of periodically poled Czochralski-grown $\mathrm{Nd}: \mathrm{Mg}: \mathrm{LiNbO} 3$ by chemical etching and X-ray microanalysis. Journal of Crystal Growth. 1997;181(1-2):160-164. doi:10.1016/S00220248(97)00246-7

7. Chiang $\mathrm{CH}$, Chen JC. Growt and-properties of Ru-doped lithium niobate crystal. Journal of Crystal Growth. 2006;294(2):323329. doi:10.1016/j.jcrysgro.2006.06.022

8. Ni DQ, Wang WY, Zhang DF, Wu X, Chen XL, Lu KQ. Nearstoichiometric $\mathrm{LiNbO} 3$ single-crystal growth by metal stripheated zone melting technique. Journal of Crystal Growth. 2004;263(1-4):421-426. doi:10.1016/j.jcrysgro.2003.12.005

9. Ballman AA. Growth of Piezoelectric and Ferroelectric Materials by the CzochraIski Technique. Journal of American Ceramic Society. 1965;48(2):112.

10. Fedulov A, Shapiro Z I, Ladyzhinskii PB. The growth of crystals of $\mathrm{LiNbO} 3, \mathrm{LiTaO} 3$ and NaNbO3 by the Czochralski method. Soviet Physics Crystallography. 1965;10:218-220.

11. Shigemastu K, Anzai Y, Morita S, Yamada M, Yokoyama H. Growth conditions of subgrain free LiNbO3 single crystals structural, electronic and optical properties showed that the samples are fit for use in high efficiency optoelectronic device applications. The characterization of magnetic properties showed that the samples were free of magnetic impurities.

\section{Acknowledgements}

This work was partially supported by the Nippon Sheet Glass Foundation for Materials Science and Engineering, the Sasagawa Postdoctoral Science Foundation Program (No. 13-001) of the Japan Science Society (JSS), Grant-in-Aid for Scientific Research (C) (No. 20550173) of Japan Society for the Promotion of Science (JSPS).

\section{Conflict of interests}

The author declares that there is no conflict of interests regarding the publication of this paper.

by Czochralski method. Japanese Journal of Applied Physics. 1987;26 Pt 1(12):1988-1996.

12. Bermudez V, Dutta PS, Serrano MD, Dieguez E. In situ poling of $\mathrm{LiNbO} 3$ bulk crystal below the Curie temperature by application of electric field after growth. Journal of Crystal Growth. 1996;169(2):409-412. doi:10.1016/S0022-0248(96)00742-7

13. Chen YL, Wen JP, Kong FS, Chen L, Zhang WL, Xu JJ, et al. Effect of Li diffusion on the composition of $\mathrm{LiNbO}_{3}$ at high temperature. Journal of Crystal Growth. 2002;242(3-4):400404.

14. Iyi N, Kitamura K, Izumi F, Yamamoto JK, Hayashi T, Asano $\mathrm{H}$, Kimura S. Comparative study of defect structures in lithium niobate with different compositions. Journal of Solid State Chemistry. 1992;101(2):340.

15. Chen J, Hu C. Quantitative analysis of $\mathrm{YIG}, \mathrm{YFeO}_{3}$ and $\mathrm{Fe}_{3} \mathrm{O}_{4}$ in LHPG-grown YIG rods. Journal of Crystal Growth. 2003;249(1-2):245-250.

16. Sarker AR, Watauchi S, Nagao M, Watanabe T, Shindo I, Tanaka I. Effects of tilting mirrors on the solid-liquid interface during floating zone growth using tilting-mirror-type infrared-heating image furnace. Journal of Crystal Growth. 2010;312:2008-2011.

17. Sarker AR, Watauchi S, Nagao M, Watanabe T, Shindo I, Tanaka I. Reduced Etch Pit Density of Rutile $\left(\mathrm{TiO}_{2}\right)$ single crystals by growth using a tilting-mirror-type infrared heating image furnace. Crystal Growth \& Design. 2010;10(9):3929-3930.

18. Sarker AR, Watauchi S, Nagao M, Watanabe T, Shindo I, Tanaka I. Effects of the diameter of rutile $\left(\mathrm{TiO}_{2}\right)$ single crystals grown using tilting-mirror-type infrared heating image furnace on solid-liquid interface and etch pit density. Journal of Crystal Growth. 2011;317(1):135-138. doi:10.1016/j.jcrysgro.2011.01.035

19. Weis RS, Gaybird TK. Lithium niobate: Summary of physical properties and crystal structure. Applied Physics A. 1985;37(4):191203.

20. Chiang CH, Chen JC. Growth and properties of Ru-doped lithium niobate crystal. Journal of Crystal Growth. 2008;310:2678.

21. Serrano MD, Bermudez V, Arizmendi L, Dieguez E. Determination of the $\mathrm{Li} / \mathrm{Nd}$ ratio in $\mathrm{LiNbO} 3$ crystals grown by Czochralski method with $\mathrm{K} 2 \mathrm{O}$ added to the melt. Journal of Crystal Growth. 2000;270(4):670-676.

22. Amdouni N, Zarrouk PH, Julien CM. Structural and electrochemical properties of $\mathrm{LiCoO}_{2}$ and $\mathrm{LiAl}_{\mathrm{y}} \mathrm{Co}_{1-\mathrm{y}} \mathrm{O}_{2}(\mathrm{y}=0.1$ and 0.2$)$ oxides: 
A comparative study of electrodes prepared by the citrate precursor. Ionics. 2003;9(1):47-55.

23. Julien C. Local cationic environment in lithium nickel-cobalt oxides used as cathode materials for lithium batteries. Solid State Ionics. 2000;136-137:887-896. doi:10.1016/S01672738(00)00503-8

24. Julien C. Local Environment in 4 Volt Cathode Materials for Li Ion Batteries. In: Julien C, Stoynov Z, editors. Materials for lithium-Ion batteries. New York: Springer; 2003. (NATO Science Series, 85). p.309-326.

25. Hirthe WM, Brittain JO. Dislocationsin rutile as revealed by the Etch-Pit Technique Journal of the American Ceramics
Society. 2006;45(1):546-554. DOI: 10.1111/j.1151-2916.1962. tb11055.x

26. KinoshitaK, Sugii K. Bridgman growth of subgrain boundary free $\mathrm{Pb}_{1-} x \mathrm{Sn} x$ Tesinglecrystals. Journal of Crystal Growth. 1985;71(2):283-288.

27. Kingegy WD. Introduction to Ceramic. New York: John Wilely \& Sons; 1967.

28. Gaudart LP, Ciomaga VC, Drados O, Guillot R, Dragoe N. Growth and characterization of $\mathrm{Li}_{\mathrm{x}} \mathrm{CoO}_{2}$ single crystals. Journal of Crystal Growth. 2011;334(1):165-169. DOI:10.1016/j. jcrysgro.2011.07.024 